\title{
To the matter of automatic stabilization of optical emission wavelength of $\mathrm{He}-\mathrm{Ne}$ laser
}

\author{
$T$ A Akhmetshina*, $G I$ Il'in, $A G$ Il'in and $R R$ Fayzullin \\ Institute of Radio Electronics and Telecommunications, KNRTU-KAI, Kazan, 10 K. Marks Street, 420111, Russian Federation
}

\begin{abstract}
The work is devoted to solving problem of long-term synchronization of optical coherent emission of a helium-neon laser in the automatic mode, which can be used in particular in portable gravitational field detectors. A block diagram of the system that solves this problem is presented, the principle of operation is described. Much attention is also paid to existing solutions, their shortcomings are described.
\end{abstract}

\section{Introduction}

At the moment, optical measurement and control systems using coherent optical emission are getting widely spread. This is due to the non-invasive and high accuracy of the measurements, which in particular reflected in the developing of systems for the gravitational field detecting.

Despite that such methods are widely spread, the long-term wavelength stabilization of the generated optical emission remains a serious problem for them. Long-term wavelength stabilization of the generated optical emission is also the important component for radio engineering data transmission systems operating under strong intersymbol interference, since they require the use of high-precision frequency standard [1-22]. Let us consider this situation in detail.

\section{Problem Statement}

In stabilized lasers systems common blocks are: frequency discriminator (internal or external), which converts the frequency deviation of the laser into a timevarying error signal; a servo system that emits and amplifies this signal, and a control element that adjusts the laser frequency in the direction of decreasing the error signal. The long-term frequency stability of the laser in such a system is no better than the zero stability of the optical discriminator, and the response of the system to short-term frequency fluctuations depends on the bandwidth of the servo system. The control element adjusts the frequency of the laser, usually by changing the length of its resonator due to the piezoelectric effect, magnetostriction, electromagnetic effect, or using an electromechanical drive.

Atomic and molecular resonances, in a number of cases the maximum transmittance of interferometers, are usually used as an optical discriminator. The main requirements for the atomic or molecular reference point is that the its frequency must be stable and reproducible at least with the accuracy required of the optical quantum standard; the relative width of the reference line should not exceed the required amount of stability more than $102-104$ times (this condition depends on the quality of the servo system).

Servo control systems operate according to the usual principle of extreme control systems, adjusting the length of the resonator so that it corresponds to the extremum on the frequency dependence of the laser radiation intensity. On practice, schemes in which the zero of the first derivative or the central zero of the third derivative of a narrow resonance is used as a discriminator are applied. The overall gain of the feedback loop depends on the magnitude of the frequency drift in the free oscillation mode, the sensitivity of the photodetector, the slope of the discriminating curve, and the required degree of frequency stability, which can reach 105 in practical circuits. The bandwidth of the servo system is determined by the spectrum of suppressed laser frequency fluctuations.

In a $3.39 \mu \mathrm{m} \mathrm{He}-\mathrm{Ne}$ laser with a methane cell, a peak output power is used as a frame to stabilize the frequency while saturating the absorption of the F2 (2) component of the $\mathrm{P}$ (7) vibrational transition of the $\mathrm{v} 3$ band of methane. A feature of the F2(2) component is the presence of a magnetic hyperfine structure of the transition, formed mainly of three strong components of different intensity with frequency intervals between adjacent components of 11.4 and $14.2 \mathrm{kHz}$. Currently, a helium-neon laser with a methane cell makes it possible to obtain the highest values of reproducibility and longterm frequency instability.

However, the implementation of stabilization of a $\mathrm{He}-\mathrm{Ne}$ laser encounters some difficulties associated with the modulation of the frequency of a laser with a small modulation index with a frequency of the order of 1-2 $\mathrm{MHz}$. In addition to modulating the frequency with

* Corresponding author: tatyana.an1995@mail.ru 
values indicated above, it is necessary to change the average frequency of the laser at low speeds within wide limits. The full cycle of tuning the average laser frequency ranges from tens of seconds to a minute. Let us dwell on the peculiarities of signal changes during the tuning of the average laser frequency over a wide range. For large offsets from the frequency of the reference peak, the amplitude of the laser output signal is practically not modulated, this is due to the small steepness of the amplification curve and small frequency deviation. As the reference frequency is approached, the modulation of the output signal increases, due to the large frequency curvature of the reference peak. At a certain point in time, the modulation coefficient of the laser radiation reaches its maximum value. The further increase in the frequency of the laser leads to a decrease in the modulation coefficient of the laser radiation. At the moment of time when the laser frequency coincides with the reference frequency, the output voltage at the output of the synchronous detector is zero, since the first harmonic of the modulating frequency is missing in the envelope spectrum of the output laser radiation. With a further increase in the laser frequency, an increase in the modulation factor of the output radiation is observed. The phase of the laser radiation envelope is opposite to the phase of the output radiation envelope at the laser frequency lower than the reference frequency of the methane cell. With a further increase in the frequency, the modulation coefficient reaches its maximum. For large values of frequency detuning relative to the reference frequency, the modulation factor is zero. The specified modulation rate zero modulation coefficient is the maximum coefficient, then again the zero modulation factor when comparing the laser frequency and the reference frequency of the methane cell and further increasing the modulation factor to the maximum value and then decreasing it to zero. Thus, the zero signal of the automatic frequency control systems is achieved when the frequencies of the laser and the reference frequency are equal and the large tune-ups relative to the reference frequency are present. This circumstance is associated with difficulties in the automatic search for a reference frequency. Therefore, the main implementations of the stabilization system are carried out as follows. The voltage on the frequency controller is manually changed and the change of the modulation factor is observed by the oscilloscope. When a certain value is reached, the automatic tracking mode is manually switched. If, as a result of switching on the automatic control system, it does not switch to the capture mode, the procedure is repeated until the capture occurs. After capture, the system enters the mode of stabilization of laser radiation. If for any reason the tracking mode is disrupted, then the laser stabilization system goes into manual search mode.

\section{Problem Solution}

We have attempted to create a fully automated laser frequency stabilization system. The block diagram of a fully automatic laser stabilization system is presented in figure. 1.

The system uses a serial commercially available laser at a wavelength of $3.39 \mu \mathrm{m}$ and a methane cell, the

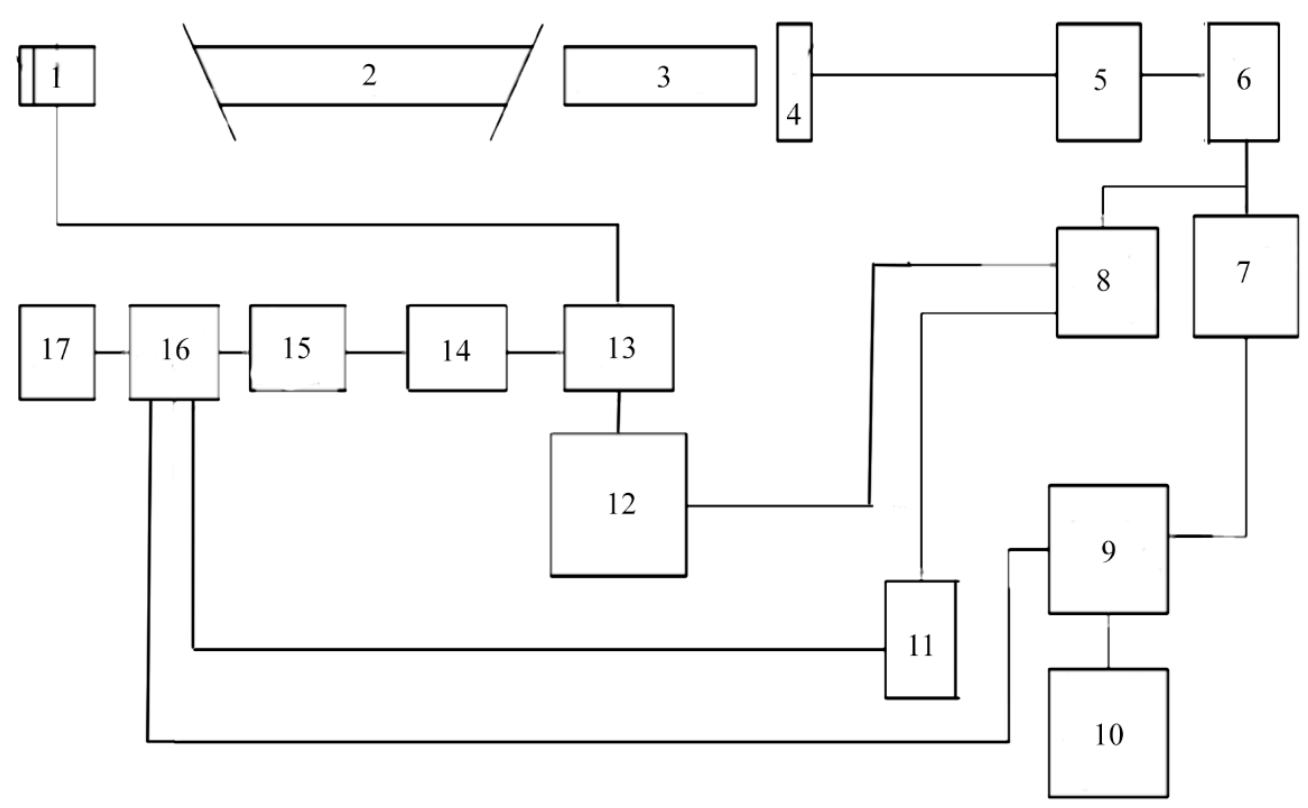

Fig. 1. Block diagram of a stabilized laser at a wavelength of $3.39 \mu \mathrm{m} .1$ is piezoelectric element with $100 \%$ mirror; 2 is active substance; 3 is methane cell; 4 is translucent mirror; 5 is photo detector; 6 is amplifier; 7 is second harmonic filter; 8 is synchronous detector; 9 is threshold device; 10 is threshold adjustment; 11 is amplifier; 12 is harmonic signal generator; 13 is voltage amplifier; 14 is DAC; 15 is pulse counter; 16 is mode switch device; 17 is clock generator. 
parameters of which are presented above. A 100\% mirror is mounted on piezoceramics, and under the action of an applied voltage can change the length of the resonator. The mirror makes two vibrations. Fast with a frequency of $1000 \mathrm{~Hz}$ and slow from the sawtooth generator, changing the average frequency of the laser. It is necessary to distinguish two modes of operation of the automatic system for stabilizing the frequency of the laser: the mode for searching for the reference frequency due to a change in the voltage on the frequency controller according to the sawtooth law (piezoelectric deaf mirror of the resonator) and frequency holding (stabilizing) mode. When the voltage on the piezoelectric mirror varies, a change in the length of the resonator and a change in the operating frequency of the laser occur. This changes the output signal that is fed to the synchronous detector, the reference frequency and phase are determined by the modulating oscillator. In the search mode, the signal from the synchronous detector is not fed into the feedback circuit and this voltage does not affect the search mode. When approaching the reference frequency after reaching the maximum modulation coefficient, the second harmonic of the modulation frequency starts to grow in the output signal. At that moment, when the voltage at the output of the synchronous detector reaches zero, the second harmonic reaches its maximum value. Therefore, the second harmonic changes from a zero value at the maximum amplitude of the first harmonic and reaches a maximum value at a zero amplitude of the first harmonic. Therefore, you can set a certain threshold level of the second harmonic, at which the system goes into tracking mode.

In order to implement this algorithm, a digital sawtooth voltage generator, consisting of a clock pulse generator, a 12-digit pulse counter, the output signals of which are fed to a digital-analog converter, was added to the automatic control system. The analog output signal is fed to an amplitude amplifier. The maximum signal applied to the piezoelectric transducer is 250 volts.

Subsequently, the system worked as follows: When a certain level of the second harmonic is reached, a control signal is generated. This signal changes the control mode of the clock generator. Depending on the sign of the output signal of the synchronous detector, the counting direction of the reversible counter of the sawtooth generator is changed. Therefore, the voltage on the piezoelectric transducer is kept within certain limits. When the hold mode is broken, the amplitude of the second-harmonic signal decreases below the threshold value. This leads to the restoration of the operating mode of the clock pulse generator, the counter goes into the pulse counting mode and the output voltage starts to change according to the sawtooth law and the cycle of the automatic control system operation repeats.

\section{Acknowledge}

This project is supported by state task of The Ministry of Education and Science of Russia № 8.5635.2017/8.9

\section{References}

1. Sh.M. Chabdarov, R.R. Faizullin, A.F. Nadeev, Post-correlation probabilistic models in the problem of signal discrimination for modern information and communication systems, Russian Aeronautics, 2, 175-180 (2014)

2. V.V. Kaduskin, R.R. Fayzullin, I.M. Lerner, An optimal algorithm for the discrimination of multielement information signals in non-gaussian CDMA channels, 18th International Conference of Young Specialists on Micro/Nanotechnologies and Electron Devices (2017)

3. I.M. Lerner, G.I. Il'in, R.R. Fayzullin, V.V.Kadushkin and A.V. Lopatina, To a question on capacity incresing o radioelectronic systems, Physics of wave processes and radiotechnical systems, 19(4), 43-49 (2016)

4. J. G. Proakis, Digital Communications, 4,928 (2000)

5. I.M. Lerner, G.I. Il'in, Limitating of capacity by selecitve systems in a channel with PSK-n-signal, 15(1), 8-12 (2017)

6. I.M. Lerner, M.I. Khairullin, G.I. Il'in, V.I. Il'in, Features of processing PSK-n-signal in conditions of ISI at linear radiopath, Nonlinear world, 15(6), 54-63 (2017)

7. I.M. Lerner, G.I. Il'in, Capacity of p-cascade tuned filter with an impact PSK-n-signal, 13th International Scientific-Technical Conference on Actual Problems of Electronics Instrument Engineering (APEIE), 1(1), 443-447 (2016)

8. I.M. Lerner, G.I. Il'in, R.R. Fayzullin, V.I. Il'in, Practical aspects of increasing the spectral efficiency of psk-n-signals due to intersymbol symbol interference, Systems of signals generating and processing in the field of on board communications (2018)

9. I.M. Lerner, G.I. Il'in, S.M. Chernyavskii, The capacity estimation of real selective chains under the influence of PSK-n-signals, 18th International Conference of Young Specialists on Micro/Nanotechnologies and Electron Devices (2017)

10. I.M. Lerner, Capacity Determination of Linear Selective Systems under the Acting of PSK-n Signal on Its Input due to the Estimation of the Error Probability per Symbol Caused by the ISI, Systems of signal synchronization, generating and processing in telecommunications, SINKHROINFO (2018)

11. V.V. Kadushkin, I.M Lerner, Determination of Occurrence Conditions for Periodically NonStationary Random Process with Establishing PSK$n$ Phase at the Output of the Linear Selective System, Systems of signal synchronization, generating and processing in telecommunications, SINKHROINFO (2018)

12. M.I. Khairullin, I.M. Lerner, V.I Il'in, Determination of the Optimal Number of Realizations of the Modulating Sequence of the PSK-n signal Necessary for Estimating the Error Probability per Symbol Caused by the ISI Produced 
by Linear Selective Systems, Systems of signal synchronization, generating and processing in telecommunications, SINKHROINFO (2018)

13. I.M. Lerner, Analytical estimation of capacity of the communicational channel with the frequency response of the resonance filter in the presence of ISI and the use of PSK-n-signal, T-Comm, 11(9), 65-73 (2017)

14. I.M. Lerner, S.M. Chernyavskii, Capacity estimation of real communicational channels with APSK-Nsignals in presence of ISI, T-Comm, 12(4),48-55 (2018)

15. I.M. Lerner, G.I. Il'in, A.G. Il'in, On the cyclostationarity of APSK-N-signals observed on the output of communication channel with intersymbol interference, Bulletin of Kazan state Technical university n.a. A.N. Tupolev, 93(3), 107117 (2018)

16. I.M. Lerner, G.I. Il'in, Numerical method for potential capacity estimation Of a communication channel with isi under utilizing of PSK-n-signal, Bulletin of Kazan state Technical university n.a. A.N. Tupolev, 93(4), 138-149 (2018)

17. I.M. Lerner, G.I. Il'in, A.G. Il'in, Probabilistic characteristics of cyclo-stationary APSK-N-signals observed at the output of the communication channel with intersymbol interference, Bulletin of Kazan state Technical university n.a. A.N. Tupolev, 93(4), 150-157 (2018)

18. I.M. Lerner, Method for capacity estimation of real communicational channels with PSK-n-signals in presence of ISI and its application, T-Comm, 11(8), $52-58(2017)$

19. I.M. Lerner, G.I. Il'in, S.M. Chernyavskii, Capacity estimation of communication channel with PSK- $n$ signals in the presence of intersymbol interference, Systems of signal synchronization, generating and processing in telecommunications, SINKHROINFO (2017)

20. M.I., Khairullin, I.M. Lerner, R.R. Faizullin, V.V. Kadushkin, M.M. Fatykhov, Selection of the initial shift for PSK signal constellation in the presence of intersymbol interference, Systems of signal synchronization, generating and processing in telecommunications, SINKHROINFO (2017)

21. S.I. Evtyanov, Transient processes in the receiveramplifier circuits, 221 (1948)

22.I.M. Lerner, G.I. Il'in, V.I. Il'in, To the Matter of Optimal Transfer Characteristics of Linear Selective Systems of Communication Channel With Memory and APSK-N signals, Systems of signal synchronization, generating and processing in telecommunications, SINKHROINFO (2019) 